\title{
Pacific
}

Journal of

Mathematics

\section{HALF-COMMUTATIVE ORTHOGONAL HOPF ALGEBRAS}

Julien Bichon And Michel Dubois-Violette

Volume 263 No. 1

May 2013 


\title{
HALF-COMMUTATIVE ORTHOGONAL HOPF ALGEBRAS
}

\author{
Julien Bichon AND Michel Dubois-Violette
}

\begin{abstract}
A half-commutative orthogonal Hopf algebra is a Hopf $*$-algebra generated by the self-adjoint coefficients of an orthogonal matrix corepresentation $v=\left(v_{i j}\right)$ that half commute in the sense that $a b c=c b a$ for any $a, b, c \in\left\{v_{i j}\right\}$. The first nontrivial such Hopf algebras were discovered by Banica and Speicher. We propose a general procedure, based on a crossed product construction, that associates to a self-transpose compact subgroup $G \subset U_{n}$ a half-commutative orthogonal Hopf algebra $\mathscr{A}_{*}(G)$. It is shown that any half-commutative orthogonal Hopf algebra arises in this way. The fusion rules of $\mathscr{A}_{*}(G)$ are expressed in term of those of $G$.
\end{abstract}

\section{Introduction}

The half-liberated orthogonal quantum group $O_{n}^{*}$ were recently discovered by Banica and Speicher [2009]. These are compact quantum groups in the sense of [Woronowicz 1987], and the corresponding Hopf $*$-algebra $A_{o}^{*}(n)$ is the universal *-algebra presented by self-adjoint generators $v_{i j}$ submitted to the relations making $v=\left(v_{i j}\right)$ an orthogonal matrix and to the half-commutation relations

$$
a b c=c b a, \quad a, b, c \in\left\{v_{i j}\right\} .
$$

The half-commutation relations arose, via Tannaka duality, from a deep study of certain tensor subcategories of the category of partitions; see [Banica and Speicher 2009]. More examples of Hopf algebras with generators satisfying the half-commutation relations were given in [Banica et al. 2010], and the classification of "easy" orthogonal Hopf algebras (which means that the tensor category of corepresentations is spanned by partitions) with generators satisfying the halfcommutation relations was very recently done in [Weber 2012].

The representation theory of $O_{n}^{*}$ was discussed in [Banica and Vergnioux 2010], where strong links with the representation theory of the unitary group $U_{n}$ were found. It followed that the fusion rules of $O_{n}^{*}$ are noncommutative if $n \geq 3$. Moreover a matrix model $A_{o}^{*}(n) \hookrightarrow M_{2}\left(\Re\left(U_{n}\right)\right)$ was found in [Banica et al. 2011].

MSC2010: 20G42, 22C05, 16T05.

Keywords: Hopf algebras, quantum groups, compact groups. 
The aim of this paper is to continue these works by a general study of what we call half-commutative orthogonal Hopf algebras: Hopf $*$-algebras generated by the self-adjoint coefficients of an orthogonal matrix corepresentation $v=\left(v_{i j}\right)$ whose coefficients satisfy the previous half-commutation relations. Our main results are as follows.

(1) To any self-transpose compact subgroup $G \subset U_{n}$ we associate a half-commutative orthogonal Hopf algebra $\mathscr{A}_{*}(G)$, with $\mathscr{A}_{*}\left(U_{n}\right) \simeq A_{o}^{*}(n)$. The Hopf algebra $\mathscr{A}_{*}(G)$ is a Hopf $*$-subalgebra of the crossed product $\mathscr{R}(G) \rtimes \mathbb{C Z}_{2}$, where the action of $\mathbb{Z}_{2}$ of $\mathscr{R}(G)$ is induced by the transposition.

(2) Conversely, any noncommutative half-commutative orthogonal Hopf algebra arises from the previous construction for some compact group $G \subset U_{n}$.

(3) The fusion rules of $\mathscr{A}_{*}(G)$ can be described in terms of those of $G$.

Therefore it follows from our study that quantum groups arising from halfcommutative orthogonal Hopf algebras are objects that are very close from classical groups. This was suggested by the representation theory results from [Banica and Vergnioux 2010], by the matrix model found in the "easy" case in [Banica et al. 2011] and by the results of [Banica et al. 2013] where it was shown that the quantum group inclusion $O_{n} \subset O_{n}^{*}$ is maximal. The techniques from [Banica et al. 2013], and especially the short five lemma for cosemisimple Hopf algebras, are used in essential way here. The use of versions of the five lemma for Hopf algebras was initiated in [Andruskiewitsch and García 2009].

The paper is organized as follows. In Section 2 we fix some notation and recall the necessary background. In Section 3 we formally introduce half-commutative orthogonal Hopf algebras, and recall the early examples from [Banica and Speicher 2009; Banica et al. 2010]. Section 4 is devoted to our main construction, which associates to a self-transpose compact subgroup $G \subset U_{n}$ a half-commutative orthogonal Hopf algebra $\mathscr{A}_{*}(G)$, and we show that any half-commutative orthogonal Hopf algebra arises in this way. At the end of the section we use our construction to propose a possible orthogonal half-liberation of the unitary group $U_{n}$. In Section 5 we describe the fusion rules of $\mathscr{A}_{*}(G)$ in terms of those of $G$.

We assume that the reader is familiar with Hopf algebras [Montgomery 1993], Hopf $*$-algebras and with the algebraic approach (via algebras of representative functions) to compact quantum groups [Dijkhuizen and Koornwinder 1994; Klimyk and Schmüdgen 1997].

\section{Preliminaries}

Classical groups. We first fix some notation. As usual, the group of complex $n \times n$ unitary matrices is denoted by $U_{n}$, while $O_{n}$ denotes the group of real orthogonal 
matrices. We denote by $\mathbb{T}$ the subgroup of $U_{n}$ consisting of scalar matrices, and by $P U_{n}$ the quotient group $U_{n} / \mathbb{T}$.

Definition 2.1. Let $G \subset U_{n}$ be a compact subgroup.

(1) We say that $G$ is self-transpose if $g^{t} \in G$ for all $g \in G$.

(2) We say that $G$ is nonreal if $G \not \subset O_{n}$, i.e., if there exists $g \in G$ with $g_{i j} \notin \mathbb{R}$, for some $i, j$.

(3) We say that $G$ is doubly nonreal if there exists $g \in G$ with $g_{i j} \overline{g_{k l}} \notin \mathbb{R}$, for some $i, j, k, l$.

Note that the subgroup $\tilde{O}_{n}=\mathbb{T} O_{n} \subset U_{n}$ (considered in [Banica et al. 2013]) is nonreal but is not doubly nonreal.

Orthogonal and unitary Hopf algebras. We next recall some definitions on the algebraic approach to compact quantum groups. We work at the level of Hopf *-algebras of representative functions. The following simple key definition arose from [Woronowicz 1987].

Definition 2.2. A unitary Hopf algebra is a $*$-algebra $A$ which is generated by elements $\left\{u_{i j} \mid 1 \leq i, j \leq n\right\}$ such that the matrices $u=\left(u_{i j}\right)$ and $\bar{u}=\left(u_{i j}^{*}\right)$ are unitaries, and such that:

(1) There is a $*$-algebra map $\Delta: A \rightarrow A \otimes A$ such that $\Delta\left(u_{i j}\right)=\sum_{k=1}^{n} u_{i k} \otimes u_{k j}$.

(2) There is a $*$-algebra map $\varepsilon: A \rightarrow \mathbb{C}$ such that $\varepsilon\left(u_{i j}\right)=\delta_{i j}$.

(3) There is a $*$-algebra map $S: A \rightarrow A^{o p}$ such that $S\left(u_{i j}\right)=u_{j i}^{*}$.

If $u_{i j}=u_{i j}^{*}$ for $1 \leq i, j \leq n$, we say that $A$ is an orthogonal Hopf algebra.

It follows that $\Delta, \varepsilon, S$ satisfy the usual Hopf $*$-algebra axioms and that $u=\left(u_{i j}\right)$ is a matrix corepresentation of $A$. Note that the definition forces that a unitary Hopf algebra is of Kac type, i.e., $S^{2}=$ id. The motivating example of unitary (resp. orthogonal) Hopf algebra is $A=\mathscr{R}(G)$, the algebra of representative functions on a compact subgroup $G \subset U_{n}$ (resp. $G \subset O_{n}$ ). Here the standard generators $u_{i j}$ are the coordinate functions which take a matrix to its $(i, j)$-entry.

In fact every commutative unitary Hopf algebra is of the form $\mathscr{R}(G)$ for some unique compact group $G \subset U_{n}$ defined by $G=\operatorname{Hom}_{*-\text { alg }}(A, \mathbb{C}$ ) (this the Hopf algebra version of the Tannaka-Krein theorem). This motivates the notation " $A=\mathscr{R}(G)$ " for any unitary (resp. orthogonal) Hopf algebra, where $G$ is a unitary (resp. orthogonal) compact quantum group.

The universal examples of unitary and orthogonal Hopf algebras are as follows [Wang 1995a]. 
Definition 2.3. The universal unitary Hopf algebra $A_{u}(n)$ is the universal $*$-algebra generated by elements $\left\{u_{i j} \mid 1 \leq i, j \leq n\right\}$ such that the matrices $u=\left(u_{i j}\right)$ and $\bar{u}=\left(u_{i j}^{*}\right)$ in $M_{n}\left(A_{u}(n)\right)$ are unitaries.

The universal orthogonal Hopf algebra $A_{o}(n)$ is the universal $*$-algebra generated by self-adjoint elements $\left\{u_{i j} \mid 1 \leq i, j \leq n\right\}$ such that the matrix $u=\left(u_{i j}\right)_{1 \leq i, j \leq n}$ in $M_{n}\left(A_{o}(n)\right)$ is orthogonal.

The existence of the Hopf $*$-algebra structural morphisms follows from the universal properties of $A_{u}(n)$ and $A_{o}(n)$. As discussed above, we use the notations $A_{u}(n)=\mathscr{R}\left(U_{n}^{+}\right)$and $A_{o}(n)=\mathscr{R}\left(O_{n}^{+}\right)$, where $U_{n}^{+}$is the free unitary quantum group and $O_{n}^{+}$is the free orthogonal quantum group.

The Hopf $*$-algebra $A_{u}(n)$ was introduced by Wang [1995a], while the Hopf algebra $A_{o}(n)$ was defined first in [Dubois-Violette and Launer 1990] under the notation $\mathscr{A}\left(I_{n}\right)$, and was then defined independently in [Wang 1995a] in the compact quantum group framework.

Exact sequences of Hopf algebras. In this subsection we recall some facts on exact sequences of Hopf algebras.

Definition 2.4. A sequence of Hopf algebra maps

$$
\mathbb{C} \rightarrow B \stackrel{i}{\rightarrow} A \stackrel{p}{\rightarrow} L \rightarrow \mathbb{C}
$$

is called preexact if $i$ is injective, $p$ is surjective and $i(B)=A^{\text {co } p}$, where

$$
A^{\text {co } p}=\{a \in A \mid(\operatorname{id} \otimes p) \Delta(a)=a \otimes 1\} .
$$

A preexact sequence as in Definition 2.4 is said to be exact [Andruskiewitsch and Devoto 1995] if in addition we have $i(B)^{+} A=\operatorname{ker}(p)=A i(B)^{+}$, where $i(B)^{+}=i(B) \cap \operatorname{ker}(\varepsilon)$. For the kind of sequences to be considered in this paper, preexactness is actually equivalent to exactness.

The following lemma, that we record for future use, is Proposition 3.2 in [Banica et al. 2013].

Lemma 2.5. Let $A$ be an orthogonal Hopf algebra with generators $u_{i j}$. Assume that we have surjective Hopf algebra map $p: A \rightarrow \mathbb{C}_{2}, u_{i j} \rightarrow \delta_{i j} g$, where $<g>=\mathbb{Z}_{2}$. Let $P_{u} A$ be the subalgebra generated by the elements $u_{i j} u_{k l}$ with the inclusion $i: P_{u} A \subset A$. Then the sequence

$$
\mathbb{C} \rightarrow P_{u} A \stackrel{i}{\rightarrow} A \stackrel{p}{\rightarrow} \mathbb{C}_{2} \rightarrow \mathbb{C}
$$

is preexact.

Exact sequences of compact groups induce exact sequences of Hopf algebras. In particular, if $G \subset U_{n}$ is a compact subgroup, we have an exact sequence of compact 
groups

$$
1 \rightarrow G \cap \mathbb{\mathbb { N }} \rightarrow G \rightarrow G / G \cap \mathbb{\mathbb { T }} \rightarrow 1,
$$

which induces an exact sequence of Hopf algebras

$$
\mathbb{C} \rightarrow \mathscr{R}(G / G \cap \mathbb{\mathbb { V }}) \rightarrow \mathscr{R}(G) \rightarrow \mathscr{R}(G \cap \mathbb{\mathbb { V }}) \rightarrow \mathbb{C}
$$

We sketch a proof of the next lemma for completeness.

Lemma 2.6. Let $G \subset U_{n}$ be a compact subgroup. Then $\mathscr{R}(G / G \cap \mathbb{\mathbb { T }})$ is the subalgebra of $\mathscr{R}(G)$ generated by the elements $u_{i j} u_{k l}^{*}, i, j, k, l \in\{1, \ldots, n\}$. Moreover, if $G=U_{n}$, then $\mathscr{R}\left(P U_{n}\right)=\mathscr{R}\left(U_{n} / \mathbb{\mathbb { T }}\right)$ is isomorphic with the commutative $*$-algebra presented by generators $w_{i j, k l}, 1 \leq i, j, k, l \leq n$ and submitted to the relations

$$
\begin{gathered}
\sum_{j=1}^{n} w_{i k, j j}=\delta_{i k}=\sum_{j=1}^{n} w_{j j, i k}, \quad w_{i j, k l}^{*}=w_{j i, l k}, \\
\sum_{k, l=1}^{n} w_{i j, k l} w_{p q, k l}^{*}=\delta_{i p} \delta_{j q} .
\end{gathered}
$$

The isomorphism is given by $w_{i j, k l} \mapsto u_{i k} u_{j l}^{*}$.

Proof. Let $p: \mathscr{R}(G) \rightarrow \mathscr{R}(G \cap \mathbb{\mathbb { V }})$ be the restriction map. It is clear $\operatorname{Ker}(p)$ is generated as a $*$-ideal by the elements $u_{i j}, i \neq j$, and $u_{i i}-u_{j j}$. Let $B$ be the subalgebra generated by the elements $u_{i j} u_{k l}^{*}$. Then $B$ is a Hopf $*$-subalgebra of $\mathscr{R}(G)$ and it is clear that $B \subset \mathscr{R}(G)^{\operatorname{co} p}$. To prove the reverse inclusion we form the Hopf algebra quotient $\mathscr{R}(G) / / B=\mathscr{R}(G) / B^{+} \mathscr{R}(G)$ and denote by $\rho: \mathscr{R}(G) \rightarrow$ $\mathscr{R}(G) / / B$ the canonical projection. It is not difficult to see that in $\mathscr{R}(G) / / B$ we have $\rho\left(u_{i j}\right)=0$ if $i \neq j$ and $\rho\left(u_{i i}\right)=\rho\left(u_{j j}\right)$ for any $i, j$. Hence there exists a Hopf $*$-algebra map $p^{\prime}: \mathscr{R}(G / \mathbb{T}) \rightarrow \mathscr{R}(G) / / B$ such that $p^{\prime} \circ p=\rho$. It follows that $\mathscr{R}(G)^{\text {co } p} \subset \mathscr{R}(G)^{\text {co } \rho}$. But since our algebras are commutative, $\mathscr{R}(G)$ is a faithfully flat $B$-module and hence by [Takeuchi 1972] (see also [Andruskiewitsch and Devoto 1995]) we have $\mathscr{R}(G)^{\text {co } \rho}=B$, and hence $\mathscr{R}(G / G \cap \mathbb{T})=\mathscr{R}(G)^{\text {co } p}=B$.

The last assertion is just the reformulation of the standard fact that $P U_{n}$ is the automorphism group of the $*$-algebra $M_{n}(\mathbb{C})$ (see, e.g., [Wang 1998]).

\section{Half-commutative Hopf algebras}

We now formally introduce half-commutative orthogonal Hopf algebras. Of course the definition of half-commutativity can be given in a general context, as follows. It was first formalized, in a probabilistic context, in [Banica et al. 2012].

Definition 3.1. Let $A$ be an algebra. We say that a family $\left(a_{i}\right)_{i \in I}$ of elements of $A$ half-commute if $a b c=c b a$ for any $a, b, c \in\left\{a_{i}, i \in I\right\}$. The algebra $A$ is said to be half-commutative if it has a family of generators that half-commute. 
At a Hopf algebra level, a reasonable definition seems to be the following one.

Definition 3.2. A half-commutative Hopf algebra is a Hopf algebra $A$ generated by the coefficients of a matrix corepresentation $v=\left(v_{i j}\right)$ whose coefficients halfcommute.

We will not study half-commutative Hopf algebras in this generality. A reason for this is that it is unclear if the half-commutativity relations outside of the orthogonal case are the natural ones in the categorical framework of [Banica and Speicher 2009]. Thus we will restrict to the following special case.

Definition 3.3. A half-commutative orthogonal Hopf algebra is a Hopf $*$-algebra $A$ generated by the self-adjoint coefficients of an orthogonal matrix corepresentation $v=\left(v_{i j}\right)$ whose coefficients half-commute.

The first example is the universal one, defined in [Banica and Speicher 2009].

Definition 3.4. The half-liberated orthogonal Hopf algebra $A_{o}^{*}(n)$ is the universal *-algebra generated by self-adjoint elements $\left\{v_{i j} \mid 1 \leq i, j \leq n\right\}$ which half-commute and such that the matrix $v=\left(v_{i j}\right)_{1 \leq i, j \leq n}$ in $M_{n}\left(A_{o}^{*}(n)\right)$ is orthogonal.

The existence of the Hopf algebra structural morphisms follows from the universal property of $A_{o}^{*}(n)$, and hence $A_{o}^{*}(n)$ is a half-commutative orthogonal Hopf algebra. We use the notation $A_{o}^{*}(n)=\mathscr{R}\left(O_{n}^{*}\right)$, where $O_{n}^{*}$ is the half-liberated orthogonal quantum group. We have $\mathscr{R}\left(O_{n}^{+}\right) \rightarrow \mathscr{R}\left(O_{n}^{*}\right) \rightarrow \mathscr{R}\left(O_{n}\right)$, i.e., $O_{n} \subset O_{n}^{*} \subset O_{n}^{+}$. At $n=2$ we have $O_{2}^{*}=O_{2}^{+}$, but for $n \geq 3$ these inclusions are strict.

Another example of half-commutative orthogonal Hopf algebra is the following one, taken from [Banica et al. 2010].

Definition 3.5. The half-liberated hyperoctahedral Hopf algebra $A_{h}^{*}(n)$ is the universal $*$-algebra generated by self-adjoint elements $\left\{v_{i j} \mid 1 \leq i, j \leq n\right\}$ which half-commute, such that $v_{i j} v_{i k}=0=v_{k i} v_{j i}$ for $k \neq j$, and such that the matrix $v=\left(v_{i j}\right)_{1 \leq i, j \leq n}$ in $M_{n}\left(A_{o}^{*}(n)\right)$ is orthogonal.

Again the existence of the Hopf algebra structural morphisms follows from the universal property of $A_{h}^{*}(n)$, and hence $A_{h}^{*}(n)$ is a half-commutative orthogonal Hopf algebra. See [Banica et al. 2010] and [Weber 2012] for further examples.

The following lemma will be an important ingredient in the proof of the structure theorem of half-commutative orthogonal Hopf algebras.

Lemma 3.6. Let $A$ be a half-commutative orthogonal Hopf algebra generated by the self-adjoint coefficients of an orthogonal matrix corepresentation $v=\left(v_{i j}\right)$ whose coefficients half-commute. Then $P_{v} A$ is a commutative Hopf $*$-subalgebra of A. If moreover A is noncommutative then there exists a Hopf $*$-algebra map 
$p: A \rightarrow \mathbb{C}_{2}$ such that for any $i, j, p\left(v_{i j}\right)=\delta_{i j} s$, where $\langle s\rangle=\mathbb{Z}_{2}$, that induces $a$ preexact sequence

$$
\mathbb{C} \rightarrow P_{v} A \stackrel{i}{\rightarrow} A \stackrel{p}{\rightarrow} \mathbb{C}_{2} \rightarrow \mathbb{C}
$$

Proof. The key observation that $P_{v} A$ is commutative is Proposition 3.2 in [Banica and Vergnioux 2010]. It is clear that $P_{v} A$ is a normal Hopf $*$-subalgebra of $A$, and hence we can form the Hopf $*$-algebra quotient $A / / P_{v} A=A / A\left(P_{v} A\right)^{+}$, with $p: A \rightarrow A / / P_{v} A$ the canonical surjection. It is not difficult to see that in $A / / P_{v} A$ we have $p\left(v_{i j}\right)=0$ if $i \neq j, p\left(v_{i i}\right)=p\left(v_{j j}\right)$ for any $i, j$ and if we put $g=p\left(v_{i i}\right)$, $g^{2}=1$. So we have to prove that $g \neq 1$. If $g=1$, then $A / / P_{v} A$ is trivial and $p=\varepsilon$. We know from [Chirvasitu 2011] that $A$ is faithfully flat as a $P_{v} A$-module (since orthogonal Hopf algebras are cosemisimple), and hence by [Schneider 1992], we have $A^{\text {co } p}=P_{v} A$. So if $g=1$ we have $A^{\text {co } p}=P_{v} A=A$ and $A$ is commutative. Thus if $A$ is noncommutative we have $g \neq 1$, the map $p$ satisfies the conditions in the statement and we have the announced exact sequence (Lemma 2.5).

Remark 3.7. The previous exact sequence is cocentral. Thus it is possible, in principle, to classify the finite-dimensional half-commutative orthogonal Hopf algebras according to the scheme used in [Bichon and Natale 2011]. The classification data will involve in particular pairs $(\Gamma, \omega)$ formed by a finite subgroup $\Gamma \subset P U_{n}$ and a cocycle $\omega \in H^{2}\left(\Gamma, \mathbb{Z}_{2}\right)$, see [Bichon and Natale 2011] for details.

\section{The main construction}

In this section we perform our main construction that associates to any self-transpose compact subgroup $G \subset U_{n}$ a half-commutative orthogonal Hopf algebra $\mathscr{A}_{*}(G)$ and we show any half-commutative orthogonal Hopf algebra arises in this way.

We begin with a well-known lemma. We give a proof for the sake of completeness.

Lemma 4.1. Let $G \subset U_{n}$ be a compact subgroup, and denote by $u_{i j}$ the coordinate functions on $G$. The following assertions are equivalent.

(1) $G$ is self-transpose.

(2) There is a unique involutive Hopf $*$-algebra automorphism $s: \mathscr{R}(G) \rightarrow \mathscr{R}(G)$ such that $s\left(u_{i j}\right)=u_{i j}^{*}$.

Moreover if $G$ is self-transpose the automorphism is nontrivial if and only $G$ is nonreal.

Proof. Assume that $G$ is self-transpose. Then we have an involutive compact group automorphism

$$
\sigma: G \rightarrow G, \quad g \mapsto\left(g^{t}\right)^{-1}=\bar{g},
$$

which induces an involutive Hopf $*$-algebra automorphism $s: \mathscr{R}(G) \rightarrow \mathscr{R}(G)$ such that $s\left(u_{i j}\right)=u_{i j}^{*}$. Uniqueness is obvious since the elements $u_{i j}$ generate $\mathscr{R}(G)$ 
as a $*$-algebra. Conversely, the existence of $s$ will ensure the existence of the automorphism $\sigma$ since $G \simeq \operatorname{Hom}_{*-\text { alg }}(\mathscr{R}(G), \mathbb{C})$, and hence $G$ will be self-transpose. The last assertion is immediate.

Definition 4.2. Let $G \subset U_{n}$ be a self-transpose nonreal compact subgroup. We denote by $\mathscr{R}(G) \rtimes \mathbb{C Z}_{2}$ the crossed product Hopf $*$-algebra associated to the involutive Hopf $*$-algebra automorphism $s$ of Lemma 4.1.

Recall that the Hopf $*$-algebra structure of $\mathscr{R}(G) \rtimes \mathbb{C Z}_{2}$ is defined as follows (see, e.g., [Klimyk and Schmüdgen 1997]).

(1) As a coalgebra, $\mathscr{R}(G) \rtimes \mathbb{C Z}_{2}=\mathscr{R}(G) \otimes \mathbb{C Z}_{2}$.

(2) We have $\left(f \otimes s^{i}\right) \cdot\left(g \otimes s^{j}\right)=f s^{i}(g) \otimes s^{i+j}$, for any $f, g \in \mathscr{R}(G)$ and $i, j \in\{0,1\}$.

(3) We have $\left(f \otimes s^{i}\right)^{*}=s^{i}(f)^{*} \otimes s^{i}$ for any $f \in \mathscr{R}(G)$ and $i \in\{0,1\}$.

(4) The antipode is given by $S\left(u_{i j} \otimes 1\right)=u_{j i}^{*} \otimes 1, S\left(u_{i j} \otimes s\right)=u_{j i} \otimes s$ (in short $S\left(f \otimes s^{i}\right)=s^{i}(S(f)) \otimes s^{i}$ for any $f \in \mathscr{R}(G)$ and $\left.i \in\{0,1\}\right)$.

For notational simplicity we denote, for $f \in \mathscr{R}(G)$, the respective elements $f \otimes 1$ and $f \otimes s$ of $\mathscr{R}(G) \rtimes \mathbb{C Z}_{2}$ by $f$ and $f s$.

Definition 4.3. Let $G \subset U_{n}$ be a self-transpose compact subgroup. We denote by $\mathscr{A}_{*}(G)$ the subalgebra of $\mathscr{R}(G) \rtimes \mathbb{C Z}_{2}$ generated by the elements $u_{i j} s$, where $i, j$ range over $\{1, \ldots, n\}$.

Proposition 4.4. Let $G \subset U_{n}$ be a self-transpose compact subgroup. Then $\mathscr{A}_{*}(G)$ is a Hopf $*$-subalgebra of $\mathscr{R}(G) \rtimes \mathbb{C Z}_{2}$, and there exists a surjective Hopf $*$-algebra morphism

$$
\pi: A_{o}^{*}(n) \rightarrow \mathscr{A}_{*}(G), \quad v_{i j} \mapsto u_{i j} s .
$$

Hence $\mathscr{A}_{*}(G)$ is a half-commutative orthogonal Hopf algebra, and is noncommutative if and only if $G$ is doubly nonreal.

Proof. We have $\left(u_{i j} s\right)^{*}=s u_{i j}^{*}=u_{i j} s$ and hence the elements $u_{i j} s$ are self-adjoint and generate a $*$-subalgebra. Moreover, using the coproduct and antipode formula, it is immediate to check that $\Delta\left(u_{i j} s\right)=\sum_{k} u_{i k} s \otimes u_{k j} s$ and $S\left(u_{i j} s\right)=u_{j i} s$, and hence $\mathscr{A}_{*}(G)$ is an orthogonal Hopf $*$-subalgebra of $\mathscr{R}(G) \rtimes \mathbb{C Z}_{2}$. We have

$$
u_{i j} s u_{k l} s u_{p q} s=u_{i j} u_{k l}^{*} u_{p q} s=u_{p q} u_{k l}^{*} u_{i j} s=u_{p q} s u_{k l} s u_{i j} s .
$$

Hence the coefficients of the orthogonal matrix $\left(u_{i j} s\right)$ half-commute, and we get our Hopf $*$-algebra map $\pi: A_{o}^{*}(n) \rightarrow \mathscr{A}_{*}(G)$. The algebra $\mathscr{A}_{*}(G)$ is commutative if and only if the elements $u_{i j} s$ pairwise commute. We have $u_{i j} s u_{k l} s=u_{i j} u_{k l}^{*}$, so $\mathscr{A}_{*}(G)$ is noncommutative if and only if there exist $i, j, k, l$ with $u_{i j} u_{k l}^{*} \neq u_{k l} u_{i j}^{*}$, which precisely means that $G$ is doubly nonreal.

The Hopf $*$-algebra $\mathscr{A}_{*}(G)$ is part of a natural preexact sequence. 
Proposition 4.5. Let $G \subset U_{n}$ be a self-transpose compact subgroup. Then there exists a Hopf $*$-algebra embedding $\mathscr{R}(G / G \cap \mathbb{T}) \hookrightarrow \mathscr{A}_{*}(G)$ and a preexact sequence

$$
\mathbb{C} \rightarrow \mathscr{R}(G / G \cap \mathbb{T}) \stackrel{j}{\rightarrow} \mathscr{A}_{*}(G) \stackrel{q}{\rightarrow} \mathbb{C}_{2} \rightarrow \mathbb{C} .
$$

Proof. The map $q$ is defined as the restriction to $\mathscr{A}_{*}(G)$ of the Hopf $*$-algebra map $\varepsilon \otimes \mathrm{id}: \mathscr{R}(G) \rtimes \mathbb{C Z}_{2} \rightarrow \mathbb{C Z}_{2}$. Hence we have $q\left(u_{i j} s\right)=\delta_{i j} s$. Let $B$ be the subalgebra of $\mathscr{A}_{*}(G)$ generated by the elements $u_{i j} s u_{k l} s=u_{i j} u_{k l}^{*}$. It is clear that $B=\mathscr{A}_{*}(G)^{\mathrm{co} q}$, and hence we have a preexact sequence

$$
\mathbb{C} \rightarrow B \stackrel{j}{\rightarrow} \mathscr{A}_{*}(G) \stackrel{q}{\rightarrow} \mathbb{C}_{2} \rightarrow \mathbb{C} .
$$

Consider now the injective Hopf algebra map $v: \mathscr{R}(G) \hookrightarrow \mathscr{R}(G) \rtimes \mathbb{C}_{2}, f \mapsto f \otimes 1$. Since $\mathscr{R}(G / G \cap \mathbb{T})=\mathscr{R}(G)^{G \cap \mathbb{T}}$ is the subalgebra generated by the elements $u_{i j} u_{k l}^{*}$ (Lemma 2.6), we have $v(\mathscr{R}(G / G \cap \mathbb{T}))=B$, and we get our preexact sequence.

We will prove (Theorem 4.7) that a noncommutative half-commutative orthogonal Hopf algebra is isomorphic to $\mathscr{A}_{*}(G)$ for some compact group $G \subset U_{n}$. Before this we first prove that the morphism in Proposition 4.4 is an isomorphism $A_{o}^{*}(n) \simeq$ $\mathscr{A}_{*}\left(U_{n}\right)$. This can be seen as a consequence of the forthcoming Theorem 4.7 , but the proof is less technical while it already well enlightens the main ideas.

Theorem 4.6. We have a Hopf $*$-algebra isomorphism $A_{o}^{*}(n) \simeq \mathscr{A}_{*}\left(U_{n}\right)$.

Proof. Let $\pi: A_{o}^{*}(n) \rightarrow \mathscr{A}_{*}\left(U_{n}\right)$ be the Hopf $*$-algebra map from Proposition 4.4, defined by $\pi\left(v_{i j}\right)=u_{i j} s$. It induces a commutative diagram of Hopf algebra maps with preexact rows

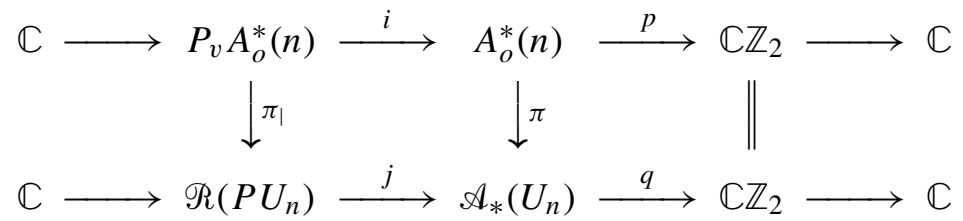

where the sequence on the top row is the one of Lemma 3.6 and the sequence on the lower row is the one of Proposition 4.5. The standard presentation of $\mathscr{R}\left(P U_{n}\right)$ (Lemma 2.6) ensures the existence of a $*$-algebra map $\mathscr{R}\left(P U_{n}\right) \rightarrow P_{v} A_{o}^{*}(n)$, $u_{i j} u_{k l}^{*} \mapsto v_{i j} v_{k l}$, which is clearly an inverse isomorphism for $\pi_{\mid}$. Thus we can invoke the short five lemma from [Banica et al. 2013, Theorem 3.4] to conclude that $\pi$ is an isomorphism.

A precursor for the previous isomorphism $A_{o}^{*}(n) \simeq \mathscr{A}_{*}\left(U_{n}\right)$ was the matrix model $A_{o}^{*}(n) \hookrightarrow M_{2}\left(\mathscr{R}\left(U_{n}\right)\right)$ found in [Banica et al. 2011, Section 8].

Theorem 4.7. Let $A$ be a noncommutative half-commutative orthogonal Hopf algebra. Then there exists a self-transpose doubly nonreal compact group $G$ with $\mathbb{T} \subset G \subset U_{n}$ such that $A \simeq \mathscr{A}_{*}(G)$. 
Proof. Let $A$ be a noncommutative half-commutative orthogonal Hopf algebra.

Step 1. We first write a convenient presentation for A. By Lemma 3.6 there exist surjective Hopf $*$-algebra maps

$$
A_{o}^{*}(n) \stackrel{f}{\rightarrow} A \stackrel{p}{\rightarrow} \mathbb{C}_{2}
$$

with $p f\left(v_{i j}\right)=\delta_{i j} s$. We denote by $V$ the comodule over $A_{o}^{*}(n)$ corresponding to the matrix $v=\left(v_{i j}\right) \in M_{n}\left(A_{o}^{*}(n)\right)$, with its standard basis $e_{1}, \ldots, e_{n}$. To any linear map $\underline{\lambda}: \mathbb{C} \rightarrow V^{\otimes m}$, with

$$
\underline{\lambda}(1)=\sum_{i_{1}, \ldots, i_{m}} \lambda\left(i_{1}, \ldots, i_{m}\right) e_{i_{1}} \otimes \cdots \otimes e_{i_{m}}
$$

we associate families $X(\underline{\lambda})$ and $X^{\prime}(\underline{\lambda})$ of elements of $A_{o}^{*}(n)$ defined by

$$
\begin{aligned}
X(\underline{\lambda}) & =\left\{\sum_{j_{1}, \ldots, j_{m}} v_{i_{1} j_{1}} \cdots v_{i_{m} j_{m}} \lambda\left(j_{1}, \ldots, j_{m}\right)-\lambda\left(i_{1}, \ldots, i_{m}\right) 1 \mid i_{1}, \ldots, i_{m} \in\{1, \ldots, n\}\right\}, \\
X^{\prime}(\underline{\lambda}) & =\left\{\sum_{j_{1}, \ldots, j_{m}} v_{j_{m} i_{m}} \cdots v_{j_{1} i_{1}} \lambda\left(j_{1}, \ldots, j_{m}\right)-\lambda\left(i_{1}, \ldots, i_{m}\right) 1 \mid i_{1}, \ldots, i_{m} \in\{1, \ldots, n\}\right\} .
\end{aligned}
$$

These elements generate a $*$-ideal in $A_{o}^{*}(n)$, which is in fact a Hopf $*$-ideal, that we denote by $I_{\underline{\lambda}}$. We also view $V$ as an $A$-comodule via $f$, and the map $\underline{\lambda}$ is a morphism of $A$-comodules if and only if $f\left(I_{\lambda}\right)=0$. Now given a family $\mathscr{C}$ of linear maps $\mathbb{C} \rightarrow V^{\otimes m}, m \in \mathbb{N}$, we denote by $I_{\mathscr{C}}$ the Hopf $*$-ideal of $A_{o}^{*}(n)$ generated by all the elements of $X(\underline{\lambda})$ and $X^{\prime}(\underline{\lambda}), \underline{\lambda} \in \mathscr{C}$. It follows from Woronowicz Tannaka-Krein duality [Woronowicz 1988] that $f$ induces an isomorphism $A_{o}^{*}(n) / I_{\mathscr{C}} \simeq A$ for a suitable set $\mathscr{C}$ of morphisms of $A$-comodules (typically $\mathscr{C}$ is a family of morphisms that generate the tensor category of corepresentations of $A$ ).

Step 2. We now construct a compact group $G$ with $\mathbb{C} \subset G \subset U_{n}$. We start with a presentation $A_{o}^{*}(n) / I_{\mathscr{C}} \simeq A$ as in Step 1 . The existence of the map $p: A \rightarrow \mathbb{C}_{2}$ implies that for $\underline{\lambda}: \mathbb{C} \rightarrow V^{\otimes m}$, if $\underline{\lambda} \neq 0$ and $\underline{\lambda} \in \mathscr{C}$, then $m$ is even (evaluate $p$ on the elements of $X(\underline{\lambda})$ ). We associate to $\underline{\lambda}: \mathbb{C} \rightarrow V^{\otimes 2 m} \in \mathscr{C}$ the following families of elements in $\mathscr{R}\left(U_{n}\right)$, where in each case $i_{1}, \ldots, i_{2 m}$ range over $\{1, \ldots, n\}$ :

$$
\begin{aligned}
& X_{1}(\underline{\lambda})=\left\{\sum_{j_{1}, \ldots, j_{2 m}} u_{i_{1} j_{1}} u_{i_{2} j_{2}}^{*} \cdots u_{i_{2 m-1} j_{2 m-1}} u_{i_{2 m} j_{2 m}}^{*} \lambda\left(j_{1}, \ldots, j_{2 m}\right)-\lambda\left(i_{1}, \ldots, i_{2 m}\right) 1\right\}, \\
& X_{1}^{\prime}(\underline{\lambda})=\left\{\sum_{j_{1}, \ldots, j_{2 m}} u_{j_{1} i_{1}}^{*} u_{j_{2} i_{2}} \cdots u_{j_{2 m-1} i_{2 m-1}}^{*} u_{j_{2 m} i_{2 m}} \lambda\left(j_{1}, \ldots, j_{2 m}\right)-\lambda\left(i_{1}, \ldots, i_{2 m}\right) 1\right\}, \\
& X_{2}(\underline{\lambda})=\left\{\sum_{j_{1}, \ldots, j_{2 m}} u_{i_{1} j_{1}}^{*} u_{i_{2} j_{2}} \cdots u_{i_{2 m-1} j_{2 m-1}}^{*} u_{i_{2 m} j_{2 m}} \lambda\left(j_{1}, \ldots, j_{2 m}\right)-\lambda\left(i_{1}, \ldots, i_{2 m}\right) 1\right\}, \\
& \left.\sum_{j_{1}, \ldots, j_{2 m}} u_{j_{1} i_{1}} u_{j_{2} i_{2}}^{*} \cdots u_{j_{2 m-1} i_{2 m-1}} u_{j_{2 m} i_{2 m}}^{*} \lambda\left(j_{1}, \ldots, j_{2 m}\right)-\lambda\left(i_{1}, \ldots, i_{2 m}\right) 1\right\} .
\end{aligned}
$$


Now denote by $J_{\mathscr{C}}$ the $*$-ideal of $\mathscr{R}\left(U_{n}\right)$ generated by the elements of $X_{1}(\underline{\lambda}), X_{1}^{\prime}(\underline{\lambda})$, $X_{2}(\underline{\lambda})$ and $X_{2}^{\prime}(\underline{\lambda})$ for all the elements $\underline{\lambda} \in \mathscr{C}$. In fact $J_{\mathscr{C}}$ is a Hopf $*$-ideal and we define $G$ to be the compact group $G \subset U_{n}$ such that $\mathscr{R}(G) \simeq \mathscr{R}\left(U_{n}\right) / J_{\mathscr{C}}$. The existence of a Hopf $*$-algebra map $\rho: \mathscr{R}(G) \rightarrow \mathbb{C} \mathbb{Z}, u_{i j} \mapsto \delta_{i j} t$, where $t$ denotes a generator of $\mathbb{Z}$, is straightforward, and thus $\mathbb{T} \subset G$. Also it is easy to check the existence of a Hopf $*$-algebra map $\mathscr{R}(G) \rightarrow \mathscr{R}(G), u_{i j} \mapsto u_{i j}^{*}$, and this shows that $G$ is self-transpose. We have, by Proposition 4.4, a Hopf $*$-algebra map $\pi: A_{o}^{*}(n) \rightarrow \mathscr{A}_{*}(G), v_{i j} \mapsto u_{i j} s$. It is a direct verification to check that $\pi$ vanishes on $I_{\mathscr{C}}$, so induces a Hopf $*$-algebra map $\bar{\pi}: A \rightarrow \mathscr{A}_{*}(G)$. We still denote by $v_{i j}$ the element $f\left(v_{i j}\right)$ in $A$. We get a commutative diagram with preexact rows

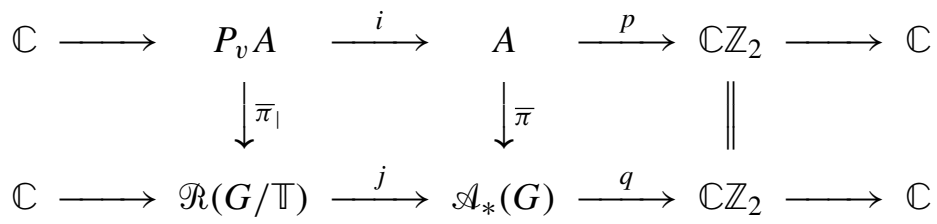

where the sequence on the top row is the one of Lemma 3.6 and the sequence on the lower row is the one of Proposition 4.5. To prove that $\bar{\pi}$ is an isomorphism, we just have, by the short five-lemma for cosemisimple Hopf algebra [Banica et al. 2013], to prove that $\bar{\pi}_{1}: P_{v} A \rightarrow \mathscr{R}(G / \mathbb{T})$ is an isomorphism. Let $J_{\mathscr{C}}^{\prime}$ be the *-ideal of $\mathscr{R}\left(P U_{n}\right)$ generated by the elements of $X_{1}(\underline{\lambda}), X_{1}^{\prime}(\underline{\lambda}), X_{2}(\underline{\lambda})$ and $X_{2}(\underline{\lambda})$ for all the elements $\underline{\lambda} \in \mathscr{C}$. It is clear, using the $\mathbb{Z}$-grading on $\mathscr{R}(G)$ induced by the inclusion $\mathbb{T} \subset G$ and the fact that $J_{\mathscr{C}}$ is generated by elements of degree zero, that $J_{\mathscr{C}}^{\prime}=J_{\mathscr{C}} \cap \mathscr{R}\left(P U_{n}\right)$, so $\mathscr{R}(G / \mathbb{T}) \simeq \mathscr{R}\left(P U_{n}\right) / J_{\mathscr{C}}^{\prime}$. But then the natural $*$-algebra map $\mathscr{R}\left(P U_{n}\right) \rightarrow P_{v} A$ (Lemma 2.6) vanishes on $J_{\mathscr{C}}^{\prime}$, and hence induces a $*$-algebra map $\mathscr{R}(G / \mathbb{T}) \rightarrow P_{v} A$, which is an inverse for $\bar{\pi}_{\mid}$. Hence $\bar{\pi}$ is an isomorphism, and the algebra $A$ being noncommutative, it follows from Proposition 4.4 that $G$ is doubly nonreal.

The proof of Theorem 4.7 also provides a method to find the compact group $G$ from the half-commutative orthogonal Hopf algebra $A$.

Example 4.8. On can check, by following the proof of Theorem 4.7, that the hyperoctahedral Hopf algebra $A_{h}^{*}(n)$ is isomorphic to $\mathscr{A}_{*}\left(K_{n}\right)$, where $K_{n}$ is the subgroup of $U_{n}$ formed by matrices having exactly one nonzero element on each column and line (with $K_{n} \simeq \mathbb{T}^{n} \rtimes S_{n}$ ).

Remark 4.9. Let $H \subset G \subset U_{n}$ be self-transpose compact subgroups. The inclusion $H \subset G$ induces a surjective Hopf $*$-algebra map $\mathscr{A}_{*}(G) \rightarrow \mathscr{A}_{*}(H)$, compatible with the exact sequence in Proposition 4.5. Thus if the inclusion $H \subset G$ induces an isomorphism $H / H \cap \mathbb{V} \simeq G / G \cap \mathbb{T}$, the short five lemma ensures that $\mathscr{A}_{*}(G) \simeq$ $\mathscr{A}_{*}(H)$. In particular, $\mathscr{A}_{*}\left(U_{n}\right) \simeq \mathscr{A}_{*}\left(S U_{n}\right)$. 
We now propose a tentative orthogonal half-liberation for the unitary group. In fact another possible half-liberation of $U_{n}$ has already been proposed in [Bhowmick et al. 2011], using the symbol $A_{u}^{*}(n)$. We shall use the notation $A_{u}^{* *}(n)$ for the object we construct, which is different from the one in [Bhowmick et al. 2011].

Example 4.10. Let $A_{u}^{* *}(n)$ be the quotient of $A_{u}(n)$ by the ideal generated by the elements

$$
a b c-c b a, \quad a, b, c, \in\left\{u_{i j}, u_{i j}^{*}\right\},
$$

Then $A_{u}^{* *}(n)$ is isomorphic with $\mathscr{A}_{*}\left(U_{2, n}\right)$, where $U_{2, n}$ is the subgroup of $U_{2 n}$ consisting of unitary matrices of the form

$$
\left(\begin{array}{rr}
A & B \\
-B & A
\end{array}\right), \quad A, B \in M_{n}(\mathbb{C})
$$

and hence is a half-commutative orthogonal Hopf algebra.

Proof. Let $\omega \in \mathbb{C}$ be a primitive fourth root of unity. We start with the probably well-known surjective Hopf $*$-algebra map

$$
\begin{aligned}
A_{o}(2 n) & \rightarrow A_{u}(n), \\
x_{i, j}, x_{n+i, n+j} & \mapsto \frac{u_{i j}+u_{i j}^{*}}{2}, i, j \in\{1, \ldots, n\}, \\
x_{n+i, j} & \mapsto \frac{u_{i j}-u_{i j}^{*}}{2 \omega}, i, j \in\{1, \ldots, n\}, \\
x_{i, n+j} & \mapsto \frac{u_{i j}^{*}-u_{i j}}{2 \omega}, i, j \in\{1, \ldots, n\},
\end{aligned}
$$

where $x_{i, j}$ denote the standard generators of $A_{o}(2 n)$. It is clear that it induces a surjective Hopf $*$-algebra map $A_{o}^{*}(2 n) \rightarrow A_{u}^{* *}(n)$, and hence $A_{u}^{* *}(n)$ is a halfcommutative orthogonal Hopf algebra.

Let $J$ be the ideal of $A_{o}^{*}(2 n)$ generated by the elements

$$
v_{i, j}-v_{n+i, n+j}, \quad v_{n+i, j}+v_{i, n+j}, i, j \in\{1, \ldots, n\}
$$

(where $v_{i, j}$ denotes the class of $x_{i j}$ in $A_{o}^{*}(n)$ ). Then $J$ is a Hopf $*$-ideal in $A_{o}^{*}(2 n)$ and the previous Hopf $*$-algebra map induces an isomorphism $A_{o}^{*}(2 n) / J \simeq A_{u}^{* *}(n)$ (the inverse sends $u_{i j}$ to $\left.x_{i j}+\omega x_{n+i, j}\right)$. Now having the presentation $A_{o}^{*}(2 n) / J \simeq A_{u}^{* *}(n)$, the proof of Theorem 4.7 yields $A_{u}^{* *}(n) \simeq \mathscr{A}_{*}\left(U_{2, n}\right)$.

\section{Representation theory}

In this section we describe the fusion rules of $\mathscr{A}_{*}(G)$ for any compact group $G$ (as usual by fusion rules we mean the set of isomorphism classes of simple comodules together with the decomposition of tensor products of simple comodules into simple 
constituents). Thanks to Theorem 4.7, this gives a description of the fusion rules of any half-commutative orthogonal Hopf algebra.

If $A$ is a cosemisimple Hopf algebra, we denote by $\operatorname{Irr}(A)$ the set of simple (irreducible) comodules over $A$. If $A=\mathscr{R}(G)$ for some compact group, then $\operatorname{Irr}(\mathscr{R}(G))=\operatorname{Irr}(G)$, the set of isomorphism classes of irreducible representations of $G$. By a slight abuse of notation, for a simple $A$-comodule $V$, we write $V \in \operatorname{Irr}(A)$.

Let $G \subset U_{n}$ be a self-transpose compact subgroup. Recall that the transposition induces an involutive compact group automorphism

$$
\sigma: G \rightarrow G, \quad g \mapsto\left(g^{t}\right)^{-1}=\bar{g} .
$$

For $V \in \operatorname{Irr}(G)$, we denote by $V^{\sigma}$ the (irreducible) representation of $G$ induced by the composition with $\sigma$. If $U$ is the fundamental $n$-dimensional representation of $G$, then $U^{\sigma} \simeq \bar{U}$.

We begin by recalling the description of the fusion rules for the crossed product $\mathscr{R}(G) \rtimes \mathbb{C Z}_{2}$. See [Wang 1995b, Theorem 3.7], for example.

Proposition 5.1. Let $G \subset U_{n}$ be a self-transpose compact subgroup. Then there is a bijection

$$
\operatorname{Irr}\left(\mathscr{R}(G) \rtimes \mathbb{C}_{2}\right) \simeq \operatorname{Irr}(G) \amalg \operatorname{Irr}(G) .
$$

More precisely, if $X \in \operatorname{Irr}\left(\mathscr{R}(G) \rtimes \mathbb{C Z}_{2}\right)$, then there exists a unique $V \in \operatorname{Irr}(G)$ with either $X \simeq V$ or $X \simeq V \otimes s$. For $V, W \in \operatorname{Irr}(G)$, we have

$$
\begin{aligned}
V \otimes(W \otimes s) & \simeq(V \otimes W) \otimes s, \\
(V \otimes s) \otimes W & \simeq\left(V \otimes W^{\sigma}\right) \otimes s, \\
(V \otimes s) \otimes(W \otimes s) & \simeq V \otimes W^{\sigma} .
\end{aligned}
$$

Proof. The description of the simple comodules follows in a straightforward manner from the fact that $\mathscr{R}(G) \rtimes \mathbb{C Z}_{2}=\mathscr{R}(G) \otimes \mathbb{C Z}_{2}$ as coalgebras. The tensor product decompositions are obtained by using character theory; see [Woronowicz 1987] or [Klimyk and Schmüdgen 1997].

Remark 5.2. If $G \subset U_{n}$ is connected and has a maximal torus $T$ of $G$ contained in $\mathbb{T}^{n}$, it follows from highest weight theory that $V^{\sigma} \simeq \bar{V}$ for any $V \in \operatorname{Irr}(G)$. We do not know if this is still true without these assumptions.

To express the fusion rules of $\mathscr{A}_{*}(G)$, we need more notation. Let $G \subset U_{n}$ be a compact subgroup, and denote by $U$ the fundamental $n$-dimensional representation of $G$. For $m \in \mathbb{Z}$, we put

$$
\operatorname{Irr}(G)_{[m]}=\left\{V \in \operatorname{Irr}(G), \quad V \subset U^{\otimes m} \otimes(U \otimes \bar{U})^{\otimes l} \text { for some } l \in \mathbb{N}\right\},
$$

where $U^{\otimes 0}=\mathbb{C}$ and for $m<0 U^{\otimes m}=\bar{U}^{\otimes-m}$. 
Now if $V \in \operatorname{Irr}(G)_{[0]}$, then $V \in \operatorname{Irr}(G / G \cap \mathbb{T}$ ) (see Lemma 2.6), and since $\mathscr{R}(G / G \cap \mathbb{T}) \subset \mathscr{A}_{*}(G)$, we get an element in $\operatorname{Irr}\left(\mathscr{A}_{*}(G)\right)$, still denoted $V$.

If $V \in \operatorname{Irr}(G)_{[1]}$, then $V \subset U \otimes(U \otimes \bar{U})^{\otimes l}$, for some $l \in \mathbb{N}$, and hence the coefficients of $V \otimes s$ belong to $\mathscr{A}_{*}(G)$. Thus we get an element of $\operatorname{Irr}\left(\mathscr{A}_{*}(G)\right)$, denoted $V s$.

Corollary 5.3. Let $G \subset U_{n}$ be a self-transpose compact subgroup. Then the map

$$
\operatorname{Irr}(G)_{[0]} \amalg \operatorname{Irr}(G)_{[1]} \rightarrow \operatorname{Irr}\left(\mathscr{A}_{*}(G)\right)
$$

given by

$$
V \mapsto \begin{cases}V & \text { if } V \in \operatorname{Irr}(G)_{[0]}, \\ V s & \text { if } V \in \operatorname{Irr}(G)_{[1]}\end{cases}
$$

is a bijection. Moreover, for $V \in \operatorname{Irr}(G)_{[0]}, W, W^{\prime} \in \operatorname{Irr}(G)_{[1]}$, we have

$$
\begin{aligned}
V \otimes W s & \simeq(V \otimes W) s, \\
W s \otimes V & \simeq\left(W \otimes V^{\sigma}\right) s, \\
W s \otimes W^{\prime} s & \simeq W \otimes W^{\prime \sigma}, \\
\overline{W s} & \simeq \bar{W}^{\sigma} s .
\end{aligned}
$$

Proof. The existence of the map follows from the discussion before the corollary, while injectivity comes from Proposition 5.1. For $V \in \operatorname{Irr}(G)_{[m]}, V^{\prime} \in \operatorname{Irr}(G)_{\left[m^{\prime}\right]}$, the simple constituents of $V \otimes V^{\prime}$ all belong to $\operatorname{Irr}(G)_{\left[m+m^{\prime}\right]}$, and that $V^{\sigma} \in \operatorname{Irr}(G)_{[-m]}$. So the isomorphisms in the statement (that all come from the isomorphisms of Proposition 5.1) yield decompositions into simple $\mathscr{A}_{*}(G)$-comodules. Thus we have a family of simple $\mathscr{A}_{*}(G)$-comodules, stable under decompositions of tensor products and conjugation, and that contains the fundamental comodule $U s$ : we conclude (e.g., from the orthogonality relations [Woronowicz 1987; Klimyk and Schmüdgen 1997]) that we have all the simple comodules.

\section{References}

[Andruskiewitsch and Devoto 1995] N. Andruskiewitsch and J. Devoto, "Extensions of Hopf algebras”, Algebra i Analiz 7:1 (1995), 22-61. In Russian; translated in St. Petersburg Math. J. 7:1 (1996), 17-52. MR 96f:16044 Zbl 0857.16032

[Andruskiewitsch and García 2009] N. Andruskiewitsch and G. A. García, "Quantum subgroups of a simple quantum group at roots of one", Compos. Math. 145:2 (2009), 476-500. MR 2010a:20102 Zbl 1236.17019

[Banica and Speicher 2009] T. Banica and R. Speicher, "Liberation of orthogonal Lie groups", Adv. Math. 222:4 (2009), 1461-1501. MR 2010j:46125 Zbl 05614878

[Banica and Vergnioux 2010] T. Banica and R. Vergnioux, "Invariants of the half-liberated orthogonal group”, Ann. Inst. Fourier (Grenoble) 60:6 (2010), 2137-2164. MR 2012f:46146 Zbl 05849155 
[Banica et al. 2010] T. Banica, S. Curran, and R. Speicher, "Classification results for easy quantum groups”, Pacific J. Math. 247:1 (2010), 1-26. MR 2011k:46099 Zbl 1208.46068

[Banica et al. 2011] T. Banica, S. Curran, and R. Speicher, "Stochastic aspects of easy quantum groups", Probab. Theory Related Fields 149:3-4 (2011), 435-462. MR 2012e:60015 Zbl 1236.60008

[Banica et al. 2012] T. Banica, S. Curran, and R. Speicher, "De Finetti theorems for easy quantum groups”, Ann. Probab. 40:1 (2012), 401-435. MR 2917777 Zbl 1242.46073

[Banica et al. 2013] T. Banica, J. Bichon, B. Collins, and S. Curran, "A maximality result for orthogonal quantum groups", Comm. Algebra 41:2 (2013), 656-665. arXiv 1106.5467

[Bhowmick et al. 2011] J. Bhowmick, F. D'Andrea, and L. Dabrowski, "Quantum isometries of the finite noncommutative geometry of the standard model”, Comm. Math. Phys. 307:1 (2011), 101-131. MR 2835874 Zbl 1236.81191

[Bichon and Natale 2011] J. Bichon and S. Natale, "Hopf algebra deformations of binary polyhedral groups”, Transform. Groups 16:2 (2011), 339-374. MR 2012g:16066 Zbl 1238.16024

[Chirvasitu 2011] A. Chirvasitu, "Cosemisimple Hopf algebras are faithfully flat over Hopf subalgebras”, preprint, 2011. arXiv 1110.6701

[Dijkhuizen and Koornwinder 1994] M. S. Dijkhuizen and T. H. Koornwinder, "CQG algebras: a direct algebraic approach to compact quantum groups", Lett. Math. Phys. 32:4 (1994), 315-330. MR 95m:16029 Zbl 0861.17005

[Dubois-Violette and Launer 1990] M. Dubois-Violette and G. Launer, "The quantum group of a nondegenerate bilinear form”, Phys. Lett. B 245:2 (1990), 175-177. MR 91j:16049 Zbl 1119.16307

[Klimyk and Schmüdgen 1997] A. Klimyk and K. Schmüdgen, Quantum groups and their representations, Springer, Berlin, 1997. MR 99f:17017 Zbl 0891.17010

[Montgomery 1993] S. Montgomery, Hopf algebras and their actions on rings, CBMS Regional Conference Series in Mathematics 82, Amer. Math. Soc., Providence, RI, 1993. MR 94i:16019 Zbl 0793.16029

[Schneider 1992] H.-J. Schneider, "Normal basis and transitivity of crossed products for Hopf algebras”, J. Algebra 152:2 (1992), 289-312. MR 93j:16032 Zbl 0789.16026

[Takeuchi 1972] M. Takeuchi, "A correspondence between Hopf ideals and sub-Hopf algebras", Manuscripta Math. 7 (1972), 251-270. MR 48 \#328 Zbl 0238.16011

[Wang 1995a] S. Wang, "Free products of compact quantum groups", Comm. Math. Phys. 167:3 (1995), 671-692. MR 95k:46104 Zbl 0838.46057

[Wang 1995b] S. Wang, "Tensor products and crossed products of compact quantum groups", Proc. London Math. Soc. (3) 71:3 (1995), 695-720. MR 96i:46074 Zbl 0837.46052

[Wang 1998] S. Wang, "Quantum symmetry groups of finite spaces", Comm. Math. Phys. 195:1 (1998), 195-211. MR 99h:58014 Zbl 1013.17008

[Weber 2012] M. Weber, "On the classification of easy quantum groups: the nonhyperoctahedral and the half-liberated case", preprint, 2012. arXiv 1201.4723

[Woronowicz 1987] S. L. Woronowicz, "Compact matrix pseudogroups", Comm. Math. Phys. 111:4 (1987), 613-665. MR 88m:46079 Zbl 0627.58034

[Woronowicz 1988] S. L. Woronowicz, "Tannaka-Kreĭn duality for compact matrix pseudogroups: twisted SU(N) groups”, Invent. Math. 93:1 (1988), 35-76. MR 90e:22033 Zbl 0664.58044

Received February 28, 2012. 
JULIEN BICHON

LABORATOIRE DE MATHÉMATIQUeS

UNIVERSITÉ BLAISE PASCAL

Campus des CÉZEauX BP 80026

63171 AubiÈre CEDEX

FRANCE

Julien.Bichon@math.univ-bpclermont.fr

Michel Dubois-Violette

LABoratoire DE PhySIQUe THÉORIQUE D' OrSAY

UNIVERSITÉ PARIS-SUD

BÂTIMENT 210

91405 ORSAY CEDEX

FRANCE

Michel.Dubois-Violette@u-psud.fr 


\title{
PACIFIC JOURNAL OF MATHEMATICS
}

\author{
msp.org/pjm
}

Founded in 1951 by E. F. Beckenbach (1906-1982) and F. Wolf (1904-1989)

\section{EDITORS}

V. S. Varadarajan (Managing Editor)

Department of Mathematics

University of California

Los Angeles, CA 90095-1555

pacific@math.ucla.edu

Paul Balmer

Department of Mathematics

University of California

Los Angeles, CA 90095-1555

balmer@math.ucla.edu

Daryl Cooper

Department of Mathematics

University of California

Santa Barbara, CA 93106-3080 cooper@math.ucsb.edu

Jiang-Hua $\mathrm{Lu}$

Department of Mathematics

The University of Hong Kong

Pokfulam Rd., Hong Kong jhlu@maths.hku.hk
Don Blasius

Department of Mathematics University of California

Los Angeles, CA 90095-1555

blasius@math.ucla.edu

Robert Finn

Department of Mathematics Stanford University

Stanford, CA 94305-2125

finn@math.stanford.edu

Sorin Popa

Department of Mathematics

University of California

Los Angeles, CA 90095-1555

popa@math.ucla.edu

Paul Yang

Department of Mathematics

Princeton University

Princeton NJ 08544-1000

yang@math.princeton.edu

\section{PRODUCTION}

Silvio Levy, Scientific Editor, production@msp.org

\section{SUPPORTING INSTITUTIONS}

ACADEMIA SINICA, TAIPEI

CALIFORNIA INST. OF TECHNOLOGY

INST. DE MATEMÁTICA PURA E APLICADA

KEIO UNIVERSITY

MATH. SCIENCES RESEARCH INSTITUTE

NEW MEXICO STATE UNIV.

OREGON STATE UNIV.

\author{
STANFORD UNIVERSITY \\ UNIV. OF BRITISH COLUMBIA \\ UNIV. OF CALIFORNIA, BERKELEY \\ UNIV. OF CALIFORNIA, DAVIS \\ UNIV. OF CALIFORNIA, LOS ANGELES \\ UNIV. OF CALIFORNIA, RIVERSIDE \\ UNIV. OF CALIFORNIA, SAN DIEGO \\ UNIV. OF CALIF., SANTA BARBARA
}

\author{
Vyjayanthi Chari \\ Department of Mathematics \\ University of California \\ Riverside, CA 92521-0135 \\ chari@math.ucr.edu \\ Kefeng Liu \\ Department of Mathematics \\ University of California \\ Los Angeles, CA 90095-1555 \\ liu@math.ucla.edu \\ Jie Qing \\ Department of Mathematics \\ University of California \\ Santa Cruz, CA 95064 \\ qing@cats.ucsc.edu
}

These supporting institutions contribute to the cost of publication of this Journal, but they are not owners or publishers and have no responsibility for its contents or policies.

See inside back cover or msp.org/pjm for submission instructions.

The subscription price for 2013 is US \$400/year for the electronic version, and \$485/year for print and electronic.

Subscriptions, requests for back issues and changes of subscribers address should be sent to Pacific Journal of Mathematics, P.O. Box 4163, Berkeley, CA 94704-0163, U.S.A. The Pacific Journal of Mathematics is indexed by Mathematical Reviews, Zentralblatt MATH, PASCAL CNRS Index, Referativnyi Zhurnal, Current Mathematical Publications and the Science Citation Index.

The Pacific Journal of Mathematics (ISSN 0030-8730) at the University of California, c/o Department of Mathematics, 798 Evans Hall \#3840, Berkeley, CA 94720-3840, is published monthly except July and August. Periodical rate postage paid at Berkeley, CA 94704, and additional mailing offices. POSTMASTER: send address changes to Pacific Journal of Mathematics, P.O. Box 4163, Berkeley, CA 94704-0163.

PJM peer review and production are managed by EditFLOW ${ }^{\circledR}$ from Mathematical Sciences Publishers.

PUBLISHED BY

mathematical sciences publishers

nonprofit scientific publishing

http://msp.org/

(C) 2013 Mathematical Sciences Publishers 


\section{PACIFIC JOURNAL OF MATHEMATICS}

Volume $263 \quad$ No. $1 \quad$ May 2013

Biharmonic hypersurfaces in complete Riemannian manifolds

Luis J. Alías, S. Carolina García-Martínez and Marco

RIGOLI

Half-commutative orthogonal Hopf algebras

JULIEN BiCHON and MiCHEL DUBOIS-ViOLETTE

Superdistributions, analytic and algebraic super Harish-Chandra pairs

Claudio CARMELI and RITA FIORESI

Orbifolds with signature $\left(0 ; k, k^{n-1}, k^{n}, k^{n}\right)$

Angel Carocca, Rubén A. Hidalgo and Rubí E.

RODRÍGUEZ

Explicit isogeny theorems for Drinfeld modules

IMIN CHEN and YOONJIN LEE

Topological pressures for $\epsilon$-stable and stable sets

XiAnfeng Ma and ERCAi Chen

Lipschitz and bilipschitz maps on Carnot groups

WILLIAM MEYERSON

Geometric inequalities in Carnot groups

FRANCESCOPAOLO MONTEFALCONE

Fixed points of endomorphisms of virtually free groups

PEDRO V. SILVA

The sharp lower bound for the first positive eigenvalue of the

Folland-Stein operator on a closed pseudohermitian $(2 n+1)$-manifold

CHIN-TUNG Wu

Remark on "Maximal functions on the unit $n$-sphere" by Peter M. Knopf 253 (1987)

HONG-QUAN LI 